7th International Workshop on Astronomy and

Relativistic Astrophysics (IWARA 2016)

International Journal of Modern Physics: Conference Series

Vol. 45 (2017) 1760072 (5 pages)

(C) The Author(s)

DOI: $10.1142 / \mathrm{S} 2010194517600722$

\title{
Light Meson Decay with Gaussian Confinement in a JKJ Decay Model
}

\author{
Eduardo S. da Veiga, Daniel T. da Silva, Mario L. L. da Silva \\ Instituto de Física e Matemática \\ Universidade Federal de Pelotas (UFPel) \\ Rua dos Ipês, Capão do Leão, 96050-500 Pelotas, RS, Brazil \\ eveiga@msn.com,neodts@gmail.com,mllsilva@gmail.com \\ Dimiter Hadjimichef \\ Instituto de Física \\ Universidade Federal do Rio Grande do Sul (UFRGS) \\ Av. Bento Gonçalves 9500, Agronomia, 91501-970 Porto Alegre, RS, Brazil \\ dimihdj@gmail.com
}

Published 15 August 2017

\begin{abstract}
A form to describe hadron strong decays is in terms of quark and gluon degrees of freedom in microscopic decay models. Initially we assume that strong decays are driven by the same inter-quark Hamiltonian which determines the spectrum, and that it incorporates gaussian confinement. An $A \rightarrow B C$ decay matrix element of the JKJ Hamiltonian involves a pair-production current matrix elements times a scattering matrix element. Diagrammatically this corresponds to an interaction between an initial line and produced pair. In this work we apply the model to the light meson sector and calculate the decay rate, comparing with the experimental values.
\end{abstract}

Keywords: Meson, Decay, Confinement.

PACS numbers: 11.15.Tk, 12.39.Jh, 13.25.-k

\section{Introduction}

Historically, the quark model calculations were used to describe the hadron spectrum. In particular in 80's this method was used by Oka and Yazaki for the calculation of the NN scattering with quark interchange. In the 90's the quark interchange techniques to meson-meson and baryon-baryon scattering were extended by Barnes and Swanson (Ref. ${ }^{1}$ ) and Hadjimichef et al (Refs. ${ }^{2-4}$ ). Recently these techniques were applied to meson decay, glueballs and other exotics.

This is an Open Access article published by World Scientific Publishing Company. It is distributed under the terms of the Creative Commons Attribution 4.0 (CC-BY) License. Further distribution of this work is permitted, provided the original work is properly cited. 


\section{E. S. da Veiga et al.}

In the present work we shall use the Fock-Tani formalism to obtain a decay amplitude for vector mesons $\rho$.

\section{The Meson in the Fock-Tani Formalism}

In the Fock-Tani formalism we can write the meson creation operators in the following form $M_{\alpha}^{\dagger}=\Phi_{\alpha}^{\mu \nu} q_{\mu}^{\dagger} \bar{q}_{\nu}^{\dagger}$, where $\Phi_{\alpha}^{\mu \nu}$ is the bound-state wave-function for twoquarks. The quark and anti-quark operators obey the following anti-commutation relations

$$
\begin{aligned}
& \left\{q_{\mu}, q_{\nu}\right\}=\left\{\bar{q}_{\mu}, \bar{q}_{\nu}\right\}=\left\{q_{\mu}, \bar{q}_{\nu}\right\}=\left\{q_{\mu}, \bar{q}_{\nu}^{\dagger}\right\}=0, \\
& \left\{q_{\mu}, q_{\nu}^{\dagger}\right\}=\left\{\bar{q}_{\mu}, \bar{q}_{\nu}^{\dagger}\right\}=\delta_{\mu \nu} .
\end{aligned}
$$

The composite meson operators satisfy non-canonical commutation relations

$$
\left[M_{\alpha}, M_{\beta}\right]=0
$$

and

$$
\left[M_{\alpha}, M_{\beta}^{\dagger}\right]=\delta_{\alpha \beta}-\Delta_{\alpha \beta}
$$

where

$$
\Delta_{\alpha \beta}=\Phi_{\alpha}^{\star \mu \gamma} \Phi_{\beta}^{\gamma \rho} q_{\rho}^{\dagger} q_{\mu}+\Phi_{\alpha}^{\star \mu \gamma} \Phi_{\beta}^{\gamma \rho} \bar{q}_{\rho}^{\dagger} \bar{q}_{\mu}
$$

The idea of the Fock-Tani formalism is to make a representation change, where the composite particles operators are described by operators that satisfy canonical commutation relations, i. e., which obey canonical relations

$$
\left[m_{\alpha}, m_{\beta}\right]=0
$$

and

$$
\left[m_{\alpha}, m_{\beta}^{\dagger}\right]=\delta_{\alpha \beta},
$$

where $m_{\alpha}^{\dagger}$ is the operators of the "ideal particles" creation. This way one can transform the composite state $|\alpha\rangle$ into an ideal state $\mid \alpha$ ). The transformations are given of the following form

$$
|\alpha\rangle \rightarrow \mid \alpha)=U^{-1}|\alpha\rangle
$$

and

$$
O \rightarrow O_{F T}=U^{-1} O U
$$

where the U operator must be unitary so that

$$
\langle\alpha \mid \alpha\rangle=(\alpha \mid \alpha)
$$

and

$$
\langle\alpha|O| \alpha\rangle=\left(\alpha\left|O_{F T}\right| \alpha\right)
$$


In the meson case for example we have

$$
\left.\left.\left.U^{-1} M_{\alpha}^{\dagger} \mid 0\right)=m_{\alpha}^{\dagger} \mid 0\right) \equiv \mid \alpha\right)
$$

where $U=\exp (t F)$ and $F$ is the generator of the meson transformation given by

$$
F=m_{\alpha}^{\dagger} \tilde{M}_{\alpha}-\tilde{M}_{\alpha}^{\dagger} m_{\alpha}
$$

with

$$
\tilde{M}_{\alpha}(t)=\sum_{i=0}^{\infty} \tilde{M}_{\alpha}^{(i)}(t)
$$

\section{The Microscopic Hamiltonian}

The JKJ Hamiltonian is given by

$$
H=\frac{1}{2} \int d^{3} x d^{3} y J_{a}^{\mu}(\vec{x}) K_{\mu \nu}^{a b}(\vec{x}-\vec{y}) J_{b}^{\nu}(\vec{y})
$$

with

$$
J_{a}^{\mu}(\vec{x})=\Psi_{\alpha}^{\dagger}(\vec{x})\left(\Gamma_{a}^{\mu}\right)_{\alpha \delta} \Psi_{\delta}(\vec{x})
$$

where

$$
\Gamma_{a}^{\mu}=A^{\mu} \otimes C_{a}
$$

and $A^{\mu}$ is related to the $\gamma^{\mu}$ matrixes (Ref. ${ }^{1}$ ). The expression of the Dirac quarks field is

$$
\Psi_{\alpha}(\vec{x})=\frac{1}{(2 \pi)^{3 / 2}} \int d^{3} k\left[u_{s \alpha}(\vec{k}) q_{s}(\vec{k})+v_{s \alpha}(\vec{k}) \bar{q}_{s}^{\dagger}(-\vec{k})\right] e^{i \vec{k} \cdot \vec{x}}
$$

After some algebraic manipulations we found

$$
H_{I}=V_{q q} q_{1}^{\dagger} \bar{q}_{2}^{\dagger} q_{3}^{\dagger} q_{4}-V_{\bar{q} \bar{q}} q_{1}^{\dagger} \bar{q}_{2}^{\dagger} \bar{q}_{3}^{\dagger} \bar{q}_{4}
$$

where

$$
\begin{aligned}
V_{q q}= & \frac{1}{2(2 \pi)^{3}} \int d^{3} k_{1} d^{3} k_{2} d^{3} k_{3} d^{3} k_{4} \delta\left(\overrightarrow{k_{3}}-\overrightarrow{k_{4}}+\overrightarrow{k_{1}}+\overrightarrow{k_{2}}\right) \\
& \times\left[D_{\nu \mu}^{b a}\left(\overrightarrow{k_{3}}-\overrightarrow{k_{4}}\right)+D_{\mu \nu}^{a b}\left(\overrightarrow{k_{1}}+\overrightarrow{k_{2}}\right)\right]\left\{\left[u_{3}^{\dagger}\left(\Gamma_{b}^{\nu}\right)_{34} u_{4}\right]\left[u_{1}^{\dagger}\left(\Gamma_{a}^{\mu}\right)_{21} v_{2}\right]\right\} \\
V_{\bar{q} \bar{q}}= & \frac{1}{2(2 \pi)^{3}} \int d^{3} k_{1} d^{3} k_{2} d^{3} k_{3} d^{3} k_{4} \delta\left(\overrightarrow{k_{3}}-\overrightarrow{k_{4}}+\overrightarrow{k_{1}}+\overrightarrow{k_{2}}\right) \\
& \times\left[D_{\nu \mu}^{b a}\left(\overrightarrow{k_{3}}-\overrightarrow{k_{4}}\right)+D_{\mu \nu}^{a b}\left(\overrightarrow{k_{1}}+\overrightarrow{k_{2}}\right)\right]\left\{\left[v_{4}^{\dagger}\left(\Gamma_{b}^{\nu}\right)_{43} v_{3}\right]\left[u_{1}^{\dagger}\left(\Gamma_{a}^{\mu}\right)_{21} v_{2}\right]\right\} .
\end{aligned}
$$


E. S. da Veiga et al.

We are interested in the calculation of $h_{f i}$. Applying the Fock-Tani transformation on the interaction Hamiltonian $H_{I}$ we have

$$
\left\langle f\left|H_{F T}\right| i\right\rangle=\delta\left(P_{\alpha}+P_{\beta}-P_{\gamma}\right) h_{f i}
$$

where,

$$
|f\rangle=m_{\beta}^{\dagger} m_{\alpha}^{\dagger}|0\rangle
$$

and

$$
|i\rangle=m_{\gamma}^{\dagger}|0\rangle
$$

Calculating the transition matrix we find

$$
\begin{aligned}
\left\langle f\left|H_{F T}\right| i\right\rangle= & V_{q q}(\mu \nu ; \sigma \rho)\left(\Phi_{\alpha}^{\mu \nu} \Phi_{\beta}^{\rho \epsilon}+\Phi_{\beta}^{\mu \nu} \Phi_{\alpha}^{\rho \epsilon}-\Phi_{\alpha}^{\mu \epsilon} \Phi_{\beta}^{\rho \nu}-\Phi_{\beta}^{\mu \epsilon} \Phi_{\alpha}^{\rho \nu}\right) \Phi_{\gamma}^{\sigma \epsilon} \\
& +V_{\bar{q} \bar{q}}(\mu \nu ; \sigma \rho)\left(\Phi_{\alpha}^{\mu \nu} \Phi_{\beta}^{\eta \rho}+\Phi_{\beta}^{\mu \nu} \Phi_{\alpha}^{\eta \rho}-\Phi_{\alpha}^{\mu \rho} \Phi_{\beta}^{\eta \nu}-\Phi_{\beta}^{\mu \rho} \Phi_{\alpha}^{\eta \nu}\right) \Phi_{\gamma}^{\eta \sigma},
\end{aligned}
$$

where the ground state wavefunction can be expressed as

$$
\Phi_{\alpha}^{\mu \nu}=\delta\left(P_{\alpha}-k_{\mu}-k_{\nu}\right) \varphi\left(k_{\mu}, k_{\nu}\right) \chi_{\alpha}^{\mu \nu} \xi_{\alpha}^{\mu \nu} \mathcal{C}_{\alpha}^{\mu \nu}
$$

with $\varphi\left(k_{\mu}, k_{\nu}\right), \chi_{\alpha}^{\mu \nu}, \xi_{\alpha}^{\mu \nu}$ and $\mathcal{C}_{\alpha}^{\mu \nu}$, being respectively space, spin, flavor and color wavefunction. For this work we consider the following process $\rho \rightarrow \pi^{+} \pi^{0}$ of the light sector, with $\rho$ meson at rest. Then $P_{\gamma}=0, P_{\beta}=-P_{\alpha}$ and only the confinement term will be considered $\left(\left(\Gamma_{a}^{\mu_{1}}\right)_{21}=T^{a} \gamma^{0}\right)$.

\section{The Confinement, Flavor and Color Factors}

In this work we consider that the confinement is gaussian

$$
D_{\mu_{1} \nu_{1}}^{a b}\left(\overrightarrow{k_{1}}+\overrightarrow{k_{2}}\right)=A e^{-B\left(\overrightarrow{k_{1}}+\overrightarrow{k_{2}}\right)^{2}}
$$

where $A$ and $B$ are the gaussian parameters. The space wave function is already a gaussian

$$
\varphi\left(k_{1}, k_{2}\right)=\left(\frac{1}{\pi b^{2}}\right)^{3 / 4} e^{-\frac{1}{8 \beta^{2}}\left(k_{1}-k_{2}\right)^{2}}
$$

The flavor factors are $f_{1}=f_{4}=-1 / \sqrt{2}, f_{2}=f_{3}=1 / \sqrt{2}$ and the color factor is $\mathcal{C}=2^{2} / 3^{3 / 2}$. Then

$$
h_{f i}=\mathcal{C}\left(f_{1} h_{f i_{1}}+f_{2} h_{f i_{2}}+f_{3} h_{f i_{3}}+f_{4} h_{f i_{4}}\right) .
$$


The full expression for $h_{f i}$ is

$$
\begin{aligned}
& h_{f i}= \mathcal{C} \\
&\left\{\frac{4}{\sqrt{2}} \frac{1}{(2 \pi)^{3}} A T^{a} T^{b} \frac{1}{2 m_{1}}\left(\frac{1}{\pi \beta^{2}}\right)^{9 / 4}\left(\frac{\pi}{\frac{1}{\beta^{2}}+B}\right)^{3 / 2}\left(\frac{\pi}{\left(\frac{1}{2 \beta^{2}}+B-\frac{B^{2} \beta^{2}}{1+B \beta^{2}}\right)}\right)^{3 / 2}\right. \\
&\left.\times\left[\frac{\left(5+16 B \beta^{2}\right)\left(P_{x}+i P_{y}\right)}{4+12 B \beta^{2}}\right] e^{-\frac{\left(1+4 B \beta^{2}\right) P^{2}}{16 \beta^{2}\left(1+3 B \beta^{2}\right)}}\right\}
\end{aligned}
$$

The decay amplitude for this process is given by

$$
\frac{d \Gamma_{\rho \rightarrow \pi^{0} \pi^{+}}}{d \Omega}=2 \pi P \frac{E_{\pi^{0}} E_{\pi^{+}}}{M_{\rho}}\left|h_{f i}\right|^{2},
$$

of where finally we obtain

$$
\begin{aligned}
\Gamma_{\rho \rightarrow \pi^{0} \pi^{+}}= & 2 \pi^{2} P^{3} \frac{E_{\pi^{0}} E_{\pi^{+}}}{M_{\rho}} A^{2} \frac{4}{3^{4}} \frac{1}{m_{1}^{2}}\left(\frac{1}{\pi \beta^{2}}\right)^{9 / 2}\left(\frac{1}{\frac{1}{\beta^{2}}+B}\right)^{3} \\
& \times\left(\frac{1}{\left(\frac{1}{2 \beta^{2}}+B-\frac{B^{2} \beta^{2}}{1+B \beta^{2}}\right)}\right)^{3}\left[\frac{\left(5+16 B \beta^{2}\right)}{4+12 B \beta^{2}}\right]^{2} e^{-\frac{2\left(1+4 B \beta^{2}\right) P^{2}}{16 \beta^{2}\left(1+3 B \beta^{2}\right)}}
\end{aligned}
$$

Adjusting the theoretical result to the experimental data of this process, we find $\Gamma=149.4 \mathrm{MeV}$ (Ref. ${ }^{5}$ ), being used for the parameters the following values: $A=450 \mathrm{GeV}, B=5.55 \mathrm{GeV}^{-2}, m=0.33 \mathrm{GeV}, M_{\pi}=0.138 \mathrm{GeV}, M_{\rho}=0.77 \mathrm{GeV}$ and $\beta \approx 0.3-0.4 \mathrm{GeV}$.

\section{Conclusions}

The Fock Tani formalism is proven appropriate not only for hadron scattering but for decay. The next step will be to apply the model for all the light sector and compare with other models and experimental data.

\section{Acknowledgments}

This work was partially financed by IFM-UFPel and IF-UFRGS, Brazil.

\section{References}

1. E. S. Ackleh, T. Barnes, and E. S. Swanson, Phys. Rev. D 54, 6811 (1996).

2. D. Hadjimichef, G. Krein, S. Szpigel, and J. S. da Veiga, Ann. of Phys. 268, 105 (1998); ibid. Phys. Lett. B 367, 317 (1996).

3. D. T. da Silva and D. Hadjimichef, J. Phys. G 30, 191 (2004).

4. D. T. da Silva, M. L. L. da Silva, J. N. de Quadros, and D. Hadjimichef, Phys. Rev. D 78, (2008) 076004.

5. K. Nakamura et al (Particle Data Group), J. Phys. G 37, 7A (2010). 